International Journal of Electrical Engineering and Technology (IJEET)

Volume 11, Issue 4, June 2020, pp. 360-367, Article ID: IJEET_11_04_040

Available online at https://iaeme.com/Home/issue/IJEET?Volume=11\&Issue $=4$

ISSN Print: 0976-6545 and ISSN Online: 0976-6553

DOI: https://doi.org/10.34218/IJEET.11.4.2020.040

(C) IAEME Publication

Scopus Indexed

\title{
QRS DETECTION FOR HEART RATE MONITORING
}

\author{
Imteyaz Ahmad \\ BIT Sindri, Dhanbad, Jharkhand, India \\ E-mail: iahmad@bitsindri.ac.in, hod.ece@bitsindri.ac.in
}

\begin{abstract}
Fast and efficient QRS detection algorithm is a essential prerequisite for analyzing disease related to heart. A study and analysis of first differentiation based QRS detection method, first and second differentiation based QRS detection method and High speed QRS detection method (Hamilton and Tompkins) was done. Algorithms based on differentiation method of QRS detection are efficient as far as computation is concerned. This method can be used for real time analysis of large dataset. On a standard dataset first differentiation method has highest detection accuracy but also the largest time error

(Average time error is $12.05 \mathrm{~ms}$ ). For the first and second differentiation based QRS detection method sensitivity and positive predictivity are 96.4 and 97.7, data error rate is 4.64 and Average Time error is $7.26 \mathrm{~ms}$. For the third method sensitivity and positive predictivity is 100 percent and data error rate is zero. Average time error is $9.13 \mathrm{~ms}$ and is having better performance as compared to differentiation based methods.
\end{abstract}

Key words: Electrocardiogram, Peak detection, Moving Average filter, QRS complex.

Cite this Article: Imteyaz Ahmad, QRS detection for Heart Rate Monitoring. International Journal of Electrical Engineering and Technology, 11(4), 2020, pp. 360-367.

https://iaeme.com/Home/issue/IJEET?Volume $=11 \&$ Issue $=4$

\section{INTRODUCTION}

The ECG is the electrical activity of the heart. It can be measured with surface electrodes on the Limb or chest. The heart rate in terms of beat per minute can be determined by counting the number of QRS pulses in a minute. The ECG wave shape is altered by cardiovascular diseases. ECG signal is contaminated by noise from many sources such as, $50 \mathrm{~Hz}$ from power line interference, EMG from muscles, motion artefact and change in the electrode skin interface. For QRS detection, $\mathrm{P}$ and $\mathrm{T}$ wave can treated as noise. Bandpass filter is used to remove the low frequency noise e.g. baseline wander and the high frequencies noise e.g. wave $50 \mathrm{~Hz}$ noise, motion artefact, EMG noise. Balda et al. 1977 proposes a defferentiation 
algorithm for QRS detection [11]which was further studied and estimated by Ahlstrom and Tompkins 1985[12].Thakore et al. 1983 propose an estimate of QRS Complex spectra an suggested that the pass band which maximize the QRS energy is approximately 5 to 15Hz[13]. Pan and Tompkin 1985 use the cascaded low pass and high pass filter to achieve 3 $\mathrm{dB}$ pass band from 5 to $11 \mathrm{~Hz}[22]$. Cuiwei et al. 1995 used a quadratic spline wavelet their conclusion was that the QRS Complex energies are in the range of 8 and 58.5 $\mathrm{Hz}$ [23].Sahambi et al. 1997 uses the first derivative of Gaussian smoothing wavelet and found that most of the QRS Complex energies are in the frequency range of 3 to $40 \mathrm{~Hz}[24]$. Benitej et al. 2000 describe a QRS detection algorithm using the properties of the hilbert transform with bandstop frequencies at 8 and $20 \mathrm{~Hz}[10]$ in order to remove muscular noise and maximize the QRS Complex respectively. Moraes et al. 2002 combined to improve QRS detector using bandpass filter between 9 and $30 \mathrm{~Hz}$ [25]. Chen and Chen 2003 introduce QRS detection algorithm based on real time moving averaging and assume that QRS frequencies are concentrated at 5-15 Hz[26]. Mahmoodabadi et al. 2005 uses Daubechies2 to detect QRS Complex in the frequency range 2-40 Hz[27]. Mohamed Algendi et al. 2010 show that 8-20 $\mathrm{Hz}$ pass band maximizes QRS and is having best signal to noise ratio[10]. Mohamed Algendi et al. 2014 suggest QRS detection algorithm for battery operated mobile devices[4]. Jinkwon Kim et al. 2016 proposes a new QRS detection algorithm which detects QRS complex efficiently having pass band frequency of band pass filter $5-25 \mathrm{~Hz}[3]$. We have to suppress $\mathrm{P}$ and $\mathrm{T}$ wave $50 \mathrm{~Hz}$ noise, motion artefact, EMG noise, baseline wander and enhance the QRS complex. We have to compare differentiation based algorithm for QRS detection and a high speed QRS detection algorithm.

\section{DATA}

ECG test signal taken from physionet database details: Record challenge/2016/training-a/[9] The ECG signal are normal, sampling frequency-2KHz, Gain: $1000 \mathrm{adu} / \mathrm{mV}$, length of signal10 seconds, storage format-16 and ADC resolution-16-bit.

\section{METHODOLOGY}

To compare differentiation based algorithms for QRS detection and a high speed QRS detection algorithm.

\subsection{First Differentiation Method for QRS Detection}

In this method a differentiator and a Moving Average filter is used [28].



Figure 1 ECG signal 


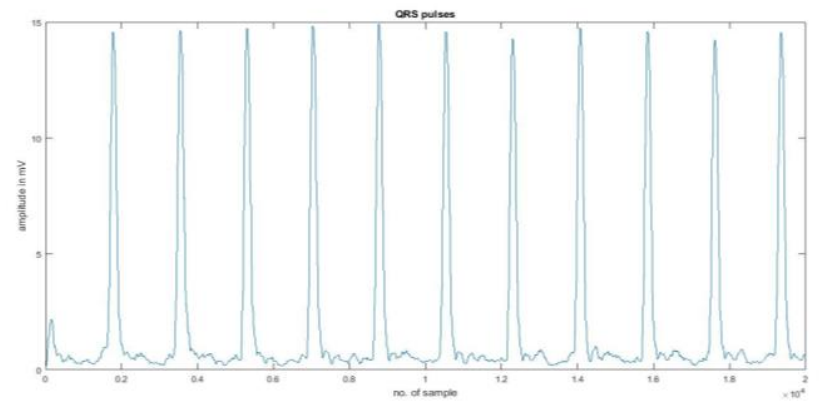

Figure 2 QRS pulses

Figure 1 in first plot illustrate three cycles of filtered version of ECG signal. The signal was filtered using 60 order band-pass FIR filter with bandwidth of $0.15-40 \mathrm{~Hz}$. The second and third plots shows the output of differentiator and the moving average filter. The fourth and fifth plots shows the QRS pulses and corresponding heart rate. It has been seen that final output contains a single peak for each QRS. Figure 2 shows QRS pulses.

\subsection{First and Second differentiation Method for QRS Detection}

QRS Complex is the largest slope in ECG signal because of contraction and relaxation of the ventricular muscles. Differentiation enhances the QRS Complex. The output of this method give a pulse for each QRS complex.[11].

The first differentiation of given signal $\mathrm{x}(\mathrm{n})$ is approximated and smoothed as:

$\mathrm{Y} 0(\mathrm{n})=\mathrm{x}(\mathrm{n})-\mathrm{x}(\mathrm{n}-2)$ and

$\mathrm{y} 0 \mathrm{sm}(\mathrm{n})=0.25 \mathrm{y} 0(\mathrm{n})+.5 \mathrm{y} 0(\mathrm{n}-1)+.25 \mathrm{y} 0(\mathrm{n}-2)$

The second differentiation of given signal $\mathrm{x}(\mathrm{n})$ is approximated and smoothed as:

$\mathrm{Y} 1(\mathrm{n})=\mathrm{x}(\mathrm{n})-2 \mathrm{x}(\mathrm{n}-2)+\mathrm{x}(\mathrm{n}-4)$

and $\mathrm{y} 1 \mathrm{sm}(\mathrm{n})=0.25 \mathrm{y} 1(\mathrm{n})+0.5 \mathrm{y} 1(\mathrm{n}-1)+0.25 \mathrm{y} 1(\mathrm{n}-2)$

The two result are weighted and combined to obtain

$\mathrm{Y} 2(\mathrm{n})=1.3 \mathrm{y} 0 \mathrm{sm}(\mathrm{n})+1.1 \mathrm{y} 1 \mathrm{sm}(\mathrm{n})$

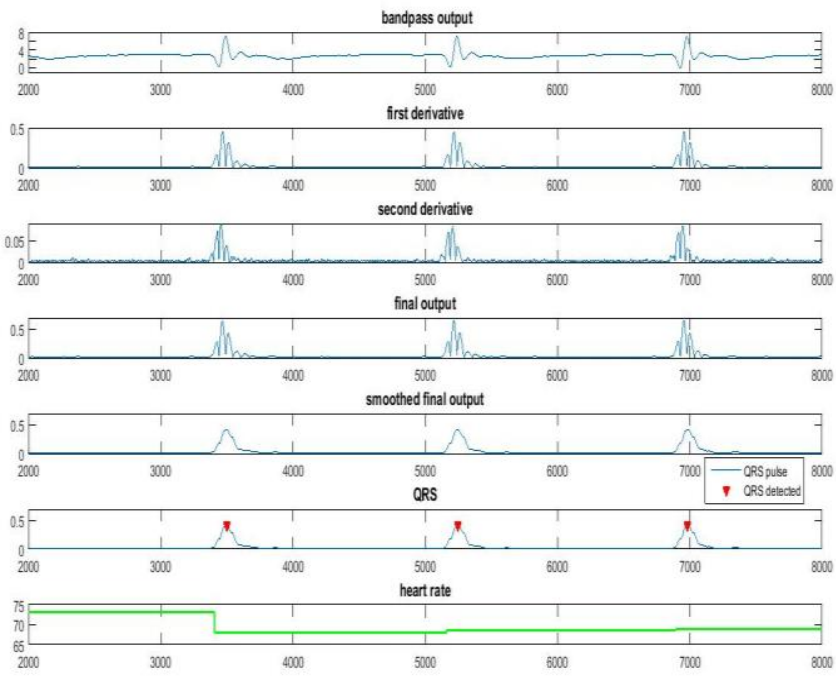

Figure 3 ECG signal

Three cycles of a filtered ECG signal is shown in first plot in Figure 3, this is output of FIR band pass filter order 60 and bandwidth of 0.15-40 Hz. The second and third plots shows 
first and second differentiation of filtered ECG. The Fourth plot shows the final output. The final output have mainly three peaks(QR,RS,S-ST baseline) these peaks can combined using moving average filter. Fifth plot shows the smoothed final output obtained by passing final output through Moving Average filter. Sixth plot shows beat detection, used to detect each ECG beat.

A heart rate monitoring is done and output is displayed in seventh plot shows corresponding heart rate. This method has the advantages of being simple to implement and produces pulses that is proportional to QRS width. The Figure 4 below shows QRS pulses.

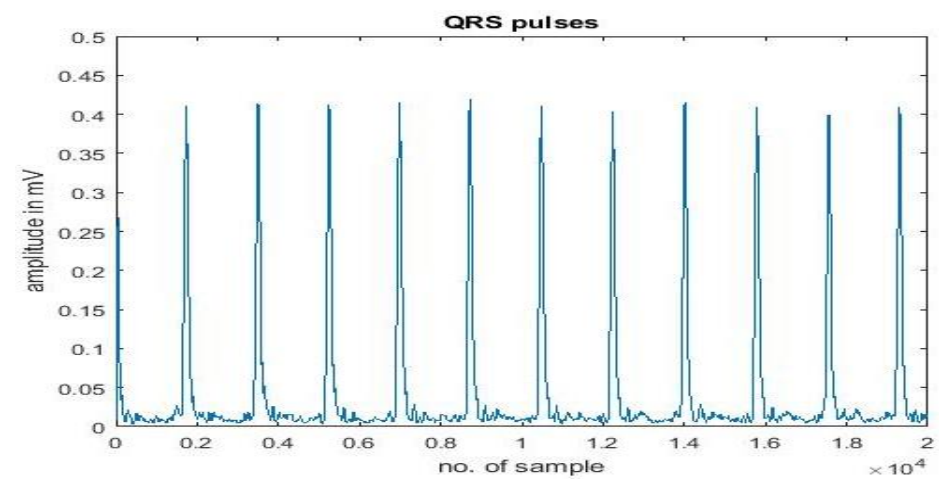

Figure 4 QRS pulses

\subsection{A High Speed QRS Detection Method}



Figure 5 QRS Detection Method

The system has five stages through which incoming ECG signal $x(n)$ is passed[30]. First a cascade of low-pass and high-pass filter is used to attenuate base wander and high frequency noise. The filter is having bandwidth of $0.15-40 \mathrm{~Hz}$ and order 60 . It is differentiated for finding the slopes that are associated with QRS complex. Each point in the sequence is squared will make all sample positive and also lead to consolidation of frequencies related to QRS complex. The squaring operation makes the result positive. High frequency components are consolidated and attenuates lower frequency component.

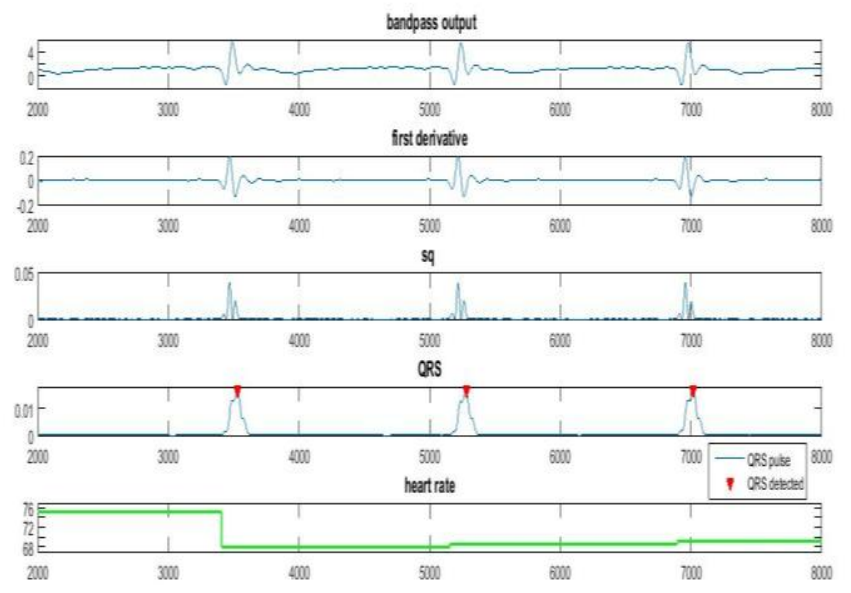

Figure 6 ECG signal 
Three cycles of a filtered ECG signal is shown in first plot in Figure 6, this is output of FIR band pass filter order 60 and bandwidth of 0.15-40 Hz. The second plots shows first derivatives of filtered ECG. The third plot shows the squared output. The squared output contains three pulses over the duration of corresponding QRS complex, can be smoothed by MA filter. Fourth plot shows the smoothed final output obtained by passing squared output through Moving Average filter. Fifth plot shows a instantaneous heart rate being monitored. The Figure 7 below shows QRS pulses.

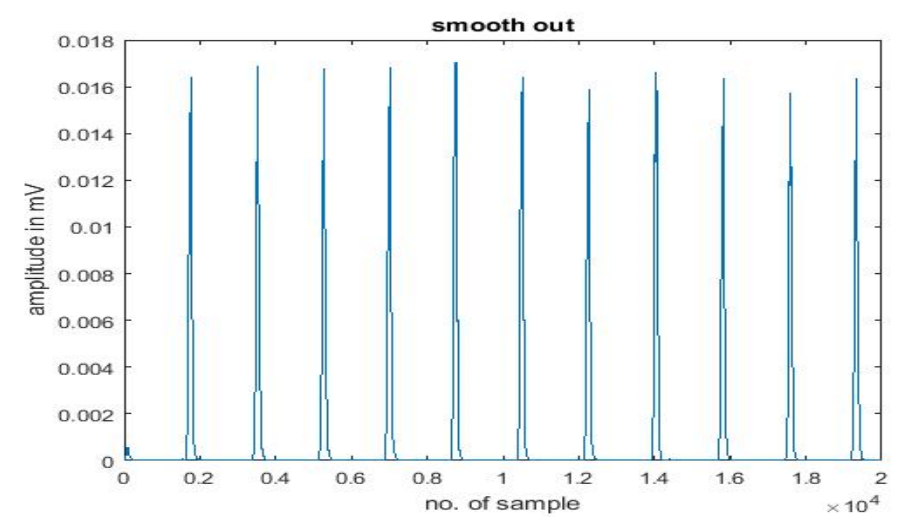

Figure 7 QRS pulses

\subsection{First differentiation Method}

Table 1 First differentiation Method

\begin{tabular}{|c|c|c|c|c|c|c|c|c|}
\hline SN & BEAT & TP & FP & FN & SE & +P & DER & Terr. ms \\
\hline $\mathrm{a} 2$ & 14 & 14 & 0 & 0 & 100 & 100 & 0 & 7.14 \\
\hline $\mathrm{a} 7$ & 13 & 13 & 0 & 0 & 100 & 100 & 0 & 8.9 \\
\hline $\mathrm{a} 9$ & 13 & 13 & 0 & 0 & 100 & 100 & 0 & 18.5 \\
\hline $\mathrm{a} 12$ & 10 & 10 & 0 & 0 & 100 & 100 & 0 & 18 \\
\hline $\mathrm{a} 16$ & 11 & 11 & 0 & 0 & 100 & 100 & 0 & 15 \\
\hline $\mathrm{a} 19$ & 11 & 11 & 0 & 0 & 100 & 100 & 0 & 4.5 \\
\hline $\mathrm{a} 27$ & 13 & 13 & 0 & 0 & 100 & 100 & 0 & 12.3 \\
\hline
\end{tabular}

\subsection{Second differentiation Method}

Table 2 Second differentiation Method

\begin{tabular}{|c|c|c|c|c|c|c|c|c|}
\hline S. no. & BEAT & TP & FP & FN & SE & + P & DER & Terr.ms \\
\hline $\mathrm{a} 2$ & 14 & 14 & 0 & 0 & 100 & 100 & 0 & 2.1 \\
\hline $\mathrm{a} 7$ & 12 & 11 & 1 & 1 & 91.6 & 91.6 & 16.6 & 12.69 \\
\hline $\mathrm{a} 9$ & 13 & 12 & 0 & 1 & 92.3 & 100 & 7.6 & 2.5 \\
\hline $\mathrm{a} 12$ & 10 & 10 & 0 & 0 & 100 & 100 & 0 & 7.5 \\
\hline $\mathrm{a} 16$ & 11 & 10 & 0 & 1 & 91.0 & 100 & 9 & 9.0 \\
\hline $\mathrm{a} 19$ & 11 & 11 & 0 & 0 & 100 & 100 & 0 & 122 \\
\hline $\mathrm{a} 27$ & 12 & 12 & 1 & 0 & 100 & 92.3 & 8.3 & 4.7 \\
\hline
\end{tabular}




\subsection{High Speed QRS Detection Algorithm (Hamilton and Tompkins)}

Table 3 High Speed QRS Detection Algorithm

\begin{tabular}{|c|c|c|c|c|c|c|c|c|}
\hline S N & BEAT & TP & FP & FN & SE & $+\mathbf{P}$ & DE & Terr. \\
\hline $\mathrm{a} 2$ & 14 & 14 & 0 & 0 & 100 & 100 & 0 & 6.78 \\
\hline $\mathrm{a} 7$ & 13 & 13 & 0 & 0 & 100 & 100 & 0 & 3.07 \\
\hline $\mathrm{a} 9$ & 13 & 13 & 0 & 0 & 100 & 100 & 0 & 6.53 \\
\hline $\mathrm{a} 12$ & 10 & 10 & 0 & 0 & 100 & 100 & 0 & 6 \\
\hline $\mathrm{a} 16$ & 11 & 11 & 0 & 0 & 100 & 100 & 0 & 5 \\
\hline $\mathrm{a} 19$ & 11 & 11 & 0 & 0 & 100 & 100 & 0 & 22.9 \\
\hline $\mathrm{a} 27$ & 13 & 13 & 0 & 0 & 100 & 100 & 0 & 13.6 \\
\hline
\end{tabular}

\section{RESULT}

Performance of QRS detection algorithm are estimated using four statistical measures: sensitivity (SE) and positive predictivity $(+\mathrm{P})$, data error rate(DER) and Average time error. Sensitivity is defined as the percentage of true beat that were correctly detected by the algorithm and Positive Predictivity is defined as the percentage of beat detections that were true beats. Data error rate(DER) can be defined as the percentage of error in beat detections that were not true beats.

Average time error $=\sum$ detected QRS time-actual QRS time/TP

Detection is said to be true positive(TP) if algorithm correctly detects the QRS Complex , false negative(FN) if algorithm fails to identify QRS Complex and false positive(FP) if the algorithm detect non- QRS complex as QRS complex.

\section{$\mathrm{SE}=\mathrm{TP} / \mathrm{TP}+\mathrm{FN}+\mathrm{P}=\mathrm{TP} / \mathrm{TP}+\mathrm{FP}$}

$\mathrm{DER}=\mathrm{FP}+\mathrm{FN} / \mathrm{BEAT}$

Average-time error $=\sum$ detected QRS time-actual QRS time/TP

Table above Shows performance of differentiation based QRS detection algorithms. Testing and analysis of algorithms made using Challenges 2016 training a database of physionet. For the first method sensitivity and positive predictivity is 100 percent and data error rate is zero. Average time error is $12.05 \mathrm{~ms}$. For the second method sensitivity and positive predictivity are 96.4 and 97.7 and data error rate is 4.64, Average Time error is $7.26 \mathrm{~ms}$. For the third method sensitivity and positive predictivity is 100 percent and data error rate is zero. Average time error is $9.13 \mathrm{~ms}$.

\section{CONCLUSION}

A study and analysis of first differentiation based QRS detection method, first and second differentiation based QRS detection method and High speed QRS detection method was done. The band pass filter used for all three method is linear FIR having bandwidth of 0.15-40 Hz. The differentiation based QRS detection methods are sensitive to high frequency noise. This problem can be overcome by High speed QRS detection method that is Hamilton and Tompkins method. For the third method sensitivity and positive predictivity is 100 percent and data error rate is zero. Average time error is $9.13 \mathrm{~ms}$ and is having better performance as compared to differentiation based methods.

\section{REFERENCES}

[1] Tae Wuk Bae and Kee Koo Kwon (2019), Efficient Real-Time R and QRS Detection Method Using a Pair of Derivative Filters and Max Filter for Portable ECG Device Appl. Sci. 2019, 9, 4128 
[2] Chieh-Li Chen and Chun-Te Chuang (2017), A QRS Detection and R Point Recognition Method for Wearable Single-Lead ECG Devices, mdpi journal/Sensors 2017, 17, 1969; doi:10.3390/s17091969

[3] JinkwonKim ,HangsikShin (2016) Simple and Robust Realtime QRS Detection Algorithm Based on Spatiotemporal Characteristic of the QRS Complex, PLOSONE|DOI:10.1371/journal.pone.0150144 March4,2016

[4] Elgendi M, Eskofier B, Dokos S, Abbott D (2014) Revisiting QRS Detection Methodologies for Portable, Wearable, Battery-Operated, and Wireless ECG Systems. PLoS ONE 9(1): e84018. doi:10.1371/journal.pone.0084018

[5] Mohamed Elgendi (2013) Fast QRS Detection with an Optimized Knowledge-Based Method: Evaluation on 11 Standard ECG Databases, PLOS ONE | www.plosone.org 1 September 2013 | Volume 8 | Issue 9 | e73557

[6] Mohamed Elgendi, Mirjam Jonkman,and Friso DeBoer (2010) frequncy bands effects on qrs detection, Conference: biosignals 2010 - Proceedings of the Third International Conference on Bio-inspired Systems and Signal Processing, Valencia, Spain, January 2023, 2010

[7] Ms,Natalia M. Arzeno, Zhi-De Deng, Chi-Sang Poon (2008), Analysis of First-Derivative Based QRS Detection Algorithms IEEE Trans Biomed Eng. 2008 February ; 55(2): 478484. doi:10.1109/TBME.2007.912658.

[8] Ren-guey lee, I-chi chou, Chien-chih lai, Ming-hsiu liu, Ming-jang chiu, (2005) A novel qrs detection algorithm applied to the analysis for heart rate variability of patients with sleep apnea, Biomed. Eng. Appl. Basis Commun. 2005.17:258-262.

[9] Goldbeerger AL, Amaral LAN, Glass L, Hausdorff JM, Ivanov PCh, Mark RG, Mietus JE, Moody GB, Peng C-K, Stanley HE.(2000) Physio Bank, Physio Toolkit, and PhysioNet: Components of a New Research Resource for Complex Physiologic S ignals. Circulation 101(23):e215-e220; (June 13).

[10] DS Benitez', PA Gaydecki, A Zaidi, AP Fitzpatrick, (2000) A New QRS Detection Algorithm Based on the Hilbert Transform, Computers in Cardiology 2000;27:379-382., 0276-6547/00 \$10.00 0 ZOO0 IEEE

[11] Balda, r. a., diller, g., deardorff, e., doue, j., \& hsieh, p. (1977). the hp ecg analysis program. trends in computer-processed electrocardiograms, 197-205.

[12] M L Ahlstrom, W J Tompkins (1985), Digital Filters for Real-Time ECG Signal Processing Using Microprocessors, IEEE Trans Biomed Eng. Sep; 32(9):708-13. doi: 10.1109/TBME.1985.325589.

[13] N.V. Thakor, Y.-S. Zhu, (1991) "Applications of adaptive filtering to ECG analysis: noise cancellation and arrhythmia detection", Biomedical Engineering IEEE Transactions on, vol. 38 , no. 8, pp. 785-794.

[14] G.M. Friesen, T.C. Jannett, M.A. Jadallah, S.L. Yates, S.R. Quint, H.T. Nagle, (1990) "A comparison of the noise sensitivity of nine QRS detection algorithms", Biomedical Engineering IEEE Transactions on, vol. 37, no. 1, pp. 85-98.

[15] P.S. Hamilton, (1996) "A comparison of adaptive and non-adaptive filters for reduction of power line interference in the ECG", Biomedical Engineering IEEE Transactions on, vol. 43, no. 1, pp. 105-109.

[16] Yong Lian, Jiang Hong Yu, (2004) "The reduction of noises in ECG signal using a frequency response masking based FIR filter", Biomedical Circuits and Systems 2004 IEEE International Workshop on, pp. S2/4-17

[17] Amin Farahabadi, Eiman Farahabadi, Hossein Rabbani, Mohammad Parsa Mahjoub, (2012) "Detection of QRS complex in electrocardiogram signal based on a combination of hilbert transform wavelet transform and adaptive thresholding", Biomedical and Health Informatics (BHI) 2012 IEEE-EMBS International Conference on, pp. 170-173. 
[18] S. Mukhopadhyay, G.C. Ray, (1998) "A new interpretation of nonlinear energy operator and its efficacy in spike detection", Biomedical Engineering IEEE Transactions on, vol. 45, no. 2, pp. 180-187.

[19] F.C. Chang, C.K. Chang, K.Y. Chi, Y.D. Lin, (2007) "Evaluation measures for adaptive PLI filters in ECG signal processing", Computers in Cardiology 2007, pp. 529-532.

[20] L.G. Herrera-Bendezu, J. Garcia, B.G. Denys, (1991) "Real-time digital filters for ECG signals: evaluation and new designs", Computers in Cardiology 1991 Proceedings, pp. 133-136.

[21] I.K. Duskalov, I.A. Dotsinsky, I.I. Christov, (1998) "Developments in ECG acquisition preprocessing parameter measurement and recording", Engineering in Medicine and Biology Magazine IEEE, vol. 17, no. 2, pp. 50-58.

[22] Jiapu pan and willis j. tompkins, (1985) A Real-Time QRS Detection Algorithm, ieee transactions on biomedical engineering, vol. bme-32, no. 3,pp-230-236.

[23] Cuiwei Li, Chongxun Zheng, and Changfeng Tai, (1995) Detection of ECG Characteristic Points Using Wavelet Transforms, ieee transactions on biomedical engineering, vol. 42, no. 1 ,pp-21-25

[24] J. S. Sahambi, S. N. Tandon and R. K. P. Bhatt, (1997) "Using wavelet transforms for ECG characterization. An on-line digital signal processing system," in IEEE Engineering in Medicine and Biology Magazine, vol. 16, no. 1, pp. 77-83, Jan.-Feb. doi: 10.1109/51.566158.

[25] JCTB Moraes, MM Freitas, FN Vilani, EV Costa, (2002) A QRS Complex Detection Algorithm using Electrocardiogram Leads, IEEE 205 Computers in Cardiology, 29:205-208

[26] H. C. Chen and S. W. Chen, (2003) "A moving average based filtering system with its application to real-time QRS detection," Computers in Cardiology, 2003, Thessaloniki Chalkidiki, Greece, pp. 585-588, doi: 10.1109/CIC.2003.1291223.

[27] S. Z. Mahmoodabadi, A. Ahmadian, and M. D. Abolhasani, (2005) "ECG Feature Extraction using Daubechies Wavelets", Proceedings of the fifth IASTED International conference on Visualization, Imaging and Image Processing, pp. 343-348.

[28] I.S.N. Murthy and M.R. Rangaraj, (1979) New concepts for PVC detection, IEEE Transactions on Biomedical Engineering,. 26(7):409-416.

[29] Ahlstrom ML, Tompkins WJ (1983) Automated high-speed analysis of Holter tapes with microcomputers. IEEE Transactions on Biomedical Engineering 30: 651-657

[30] P. S. Hamilton and W. J. Tompkins, (1986) "Quantitative Investigation of QRS Detection Rules Using the MIT/BIH Arrhythmia Database," in IEEE Transactions on Biomedical Engineering, vol. BME-33, no. 12, pp. 1157-1165, Dec. doi: 10.1109/TBME.1986.325695.

[31] Nilesh Parihar, Dr. V. S. Chouhan, (2012) Extraction of QRS Complexes Using Automated Bayesian Regularization Neural Network, International Journal of Advanced Research in Engineering and Technology, 3(2), pp. 37-42.

[32] Manju S Hadagali, Mrs. Laxmi Pai, (2014) Performance Analysis of ECG QRS Complex Detection Using Morphological Operators, International Journal of Electronics and Communication Engineering \& Technology, 5(8), pp. 200-207 